\title{
ARTICLE OPEN Scalable repeater architectures for multi-party states
}

\author{
V. V. Kuzmin $\left(\mathbb{D}^{1,2 *}\right.$, D. V. Vasilyev $\mathbb{D}^{1,2}$, N. Sangouard ${ }^{3}$, W. Dür $\mathbb{I D}^{4}$ and C. A. Muschik ${ }^{4,5}$
}

The vision to develop quantum networks entails multi-user applications, which require the generation of long-distance multi-party entangled states. The current rapid experimental progress in building prototype-networks calls for new design concepts to guide future developments. Here we describe an experimentally feasible scheme implementing a two-dimensional repeater network for robust distribution of three-party entangled states of GHZ type in the presence of excitation losses and detector dark counts - the main sources of errors in real-world hardware. Our approach is based on atomic or solid state ensembles and employs built-in error filtering mechanisms peculiar to intrinsically two-dimensional networks. This allows us to overcome the performance limitation of conventional one-dimensional ensemble-based networks distributing multi-party entangled states and provides an efficient design for future experiments with a clear perspective in terms of scalability.

npj Quantum Information (2019)5:115; https://doi.org/10.1038/s41534-019-0230-3

\section{INTRODUCTION}

The development of quantum networks holds the promise to realize quantum technologies such as secure communication schemes, distributed quantum computing, ${ }^{2}$ and metrological applications. ${ }^{3-6}$ This prospect resulted in intense efforts to build prototype-networks with few nodes. The creation of long-range bipartite entangled states has been demonstrated ${ }^{7-17}$ and multiparty entangled states shared between several remote parties can be realized in the near-future. The ability to distribute such multiparty entangled states over long-distances ${ }^{18}$ will be an essential prerequisite for future quantum networks ${ }^{19-24}$ consisting of large two-dimensional (2D) structures that allow for multi-user applications. This need and the current experimental possibilities raise the question which type of architecture can be used to distribute long-range multi-party entanglement in a practical and scalable fashion. We address this problem from an implementationoriented point of view and propose a networking scheme that allows for a robust and efficient realization with current and nearfuture experimental means.

Future quantum networks will require different types of multiparty entangled states suitable for different tasks. We concentrate on distributing three-party GHZ states, ${ }^{25}$ e.g. $(|001\rangle+|110\rangle) / \sqrt{2}$, between remote parties, that can be used for clock synchronization, $^{3}$ quantum secret sharing ${ }^{26,27}$ quantum secret voting, ${ }^{28}$ or for fundamental tests of nature. ${ }^{29}$ A key challenge for distributing quantum states over long-distances is the fact that losses and decoherence scale exponentially in the distance. ${ }^{30,31}$ To solve this problem, quantum repeaters ${ }^{32-34}$ have been introduced, which however are inherently one-dimensional (1D) schemes aiming at generating a bipartite entangled state connecting two remote parties. Therefore, an intrinsically 2D network can be preferable for a multipartite entanglement distribution as it was shown ${ }^{35}$ to have a higher error threshold than a 1D counterpart under assumptions of a generic noise model and full Bell state analysis (or a universal gate set) availability.

In this work, we propose an implementation-oriented 2D repeater scheme that, in contrast to, ${ }^{35}$ (i) can be realized using a constrained set of quantum operations available in atomic or solid state ensembles and (ii) has a mechanism to mitigate an excitation loss error and detector dark counts - major imperfections for this type of system. The resulting robust architecture is custom-tailored to implementations based on atomic or solid state ensembles, as these systems are promising for realizing long-lived quantum memories. ${ }^{36,37}$ While atomic ensembles allow a limited set of operations for processing quantum information, record coherence lifetimes $\left(2 T_{\text {coh }}\right)$ up to $6 \mathrm{~h}$ have been observed in rare-earth doped crystals. ${ }^{38}$ This makes them particularly promising for entanglement distribution over continental distances using repeater schemes which inevitably requires memory times corresponding to the duration needed for classical communication between remote nodes.

Our scheme is inspired by the seminal DLCZ proposal for generating Bell pairs between two nodes, ${ }^{39}$ and requires optical cavities with good cooperativities, linear optical elements, and photodetectors. GHZ states are first generated over moderate distances and then merged to form $\mathrm{GHZ}$ states connecting increasingly remote parties [see inset in Fig. 1a and Fig. 2a]. Similar to the DLCZ proposal, our protocol does not require a universal gate set or a full Bell-state analysis. However, unlike the DLCZ scheme, the new protocol suppresses the propagation of so-called vacuum and multi-excitation errors. These errors are resulted from excitation loss or detection of a dark count during the generation of the elementary state or merging operation and lead to the preparation of a state containing more/fewer excitations than expected. In the DLCZ scheme, the vacuum and multi-excitation errors freely propagate decreasing fidelity dramatically with the increase of nesting level and therefore making the scheme not scalable (Sangouard et al. ${ }^{34}$ and Sec. A1 of Supplemental Material). In comparison, our 2D scheme results in a truly scalable architecture. As shown in Fig. 1 and explained below, this feature allows our repeater scheme to cover longer distances than networks based on combining conventional (one-dimensional) quantum links.

${ }^{1}$ Center for Quantum Physics, Faculty of Mathematics, Computer Science and Physics, University of Innsbruck, A-6020 Innsbruck, Austria. ${ }^{2}$ Institute for Quantum Optics and Quantum Information of the Austrian Academy of Sciences, A-6020 Innsbruck, Austria. ${ }^{3}$ Department of Physics, University of Basel, 4056 Basel, Switzerland. ${ }^{4}$ Institute for Theoretical Physics, University of Innsbruck, A-6020 Innsbruck, Austria. ${ }^{5}$ Institute for Quantum Computing and Department of Physics and Astronomy, University of Waterloo, Waterloo, ON N2L 3G1, Canada. *email: viacheslav.kuzmin@uibk.ac.at 


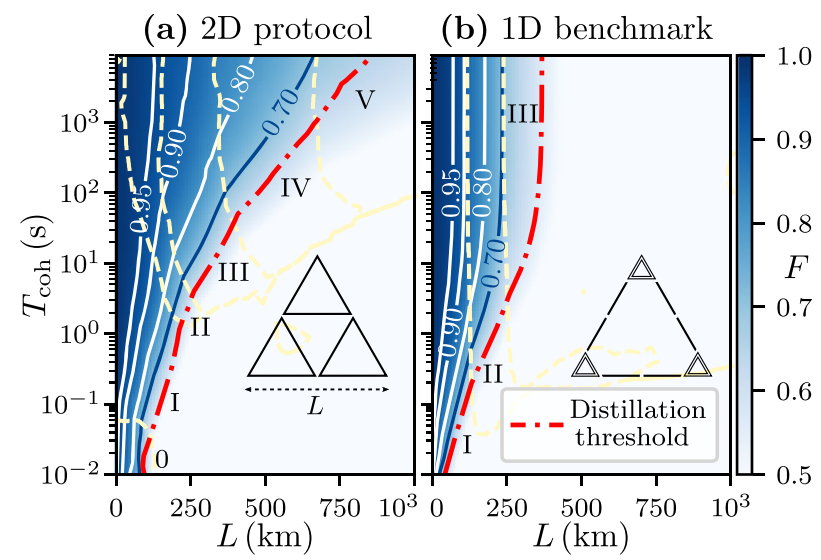

Fig. 1 Generation of long-distance GHZ states. a Generation with the 2D scheme shown in inset and Fig. 2 and $\mathbf{b}$ with the 1D benchmark (see inset and text). Both panels display the fidelity $F$ versus the memory excitation lifetime $T_{\text {coh }}$ and the covered distance $L$ for the parameters specified in Methods section. Red dash-dotted lines indicate the threshold beyond which entanglement distillation is impossible (see Supplemental Material, Sec. D). Dashed lines separate network structures with a different optimal number of nesting levels (indicated with roman numbers).

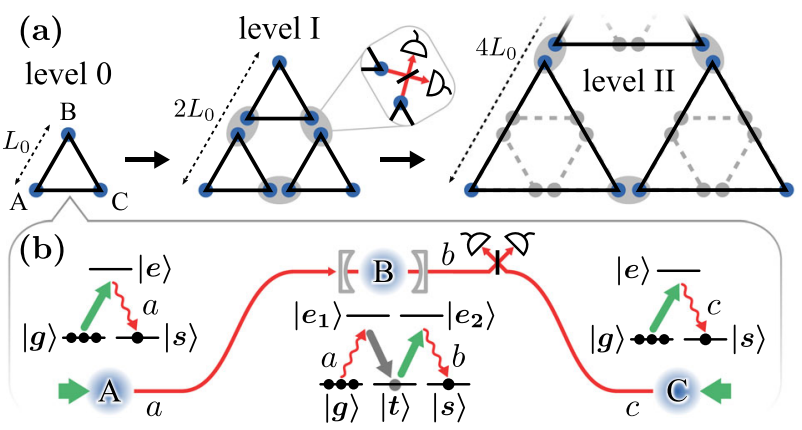

Fig. 2 2D repeater scheme. a Merging of GHZ states of three elementary segments spanning the length $L_{0}$ into a $\mathrm{GHZ}$ state of a segment covering the length $2 L_{0}$, which are further merged to a segment of the length $4 L_{0}$. Blue circles represent ensembles, and triangles depict network segments. The merging is realized by three swapping operations (see text) that are shown as gray ellipses. b Generation of the GHZ states at the elementary level. At nodes A and $C$, a $\Lambda$-type level structure is used allowing for standard DLCZtype write-in and read-out operations. ${ }^{39}$ Node $B$ employs an ensemble with a double- $\Lambda$ configuration placed in a cavity (see Supplemental Material, Sec. G).

\section{RESULTS}

Scheme

We propose a nested structure of quantum network ${ }^{35}$ consisting of segments in three-party entangled states of increasing size as shown in Fig. 2a. The scheme requires the ability to generate $\mathrm{GHZ}$ states of elementary segments spanning a length $L_{0}$, and generalized entanglement swapping procedure to merge the GHZ states doubling the covered distance. We detail the generation and swapping operations separately in the next two paragraphs.

The generation of $\mathrm{GHZ}$ states at the elementary level is illustrated in Fig. 2b. We consider two types of ensembles: (i) ensembles with a $\Lambda$-level scheme at nodes $A$ and $C$, allowing for

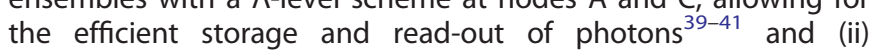
ensembles with a double- $\Lambda$ configuration placed in a cavity at node $\mathrm{B}$. Information is encoded in the absence or presence of a collective spin excitation in the ensembles, i.e. in the logical states $|0\rangle=\left|g_{1} g_{2} \ldots g_{N}\right\rangle$ and $|1\rangle=\frac{1}{\sqrt{N}} \sum_{i=1}^{N}\left|g_{1} g_{2} \ldots s_{i} \ldots g_{N}\right\rangle$, where $N$ is the number of emitters per ensemble and $|g\rangle,|s\rangle$ denote ground state levels of the emitters [see Fig. 2b]. Ensembles $A$ and $C$ are driven by weak coherent laser pulses resulting in entangled states of the ensembles and the corresponding forward-scattered photons, ${ }^{39}$ $\left|0_{A(C)} 0_{a(c)}\right\rangle+\epsilon_{a(c)}\left|1_{A(C)} 1_{a(c)}\right\rangle+\mathcal{O}\left(\epsilon_{a(c)}^{2}\right), \epsilon_{a(c)} \ll 1$ (the role of the parameter $\epsilon$ is explained in Sec. B of Supplemental Material, our analysis includes also higher order terms). The capital and lowercase subscripts refer to the states of the ensemble and the corresponding emitted light field. Node $B$ performs the gate operation $\left|1_{a} 0_{B} 0_{b}\right\rangle \rightarrow\left|0_{a} 1_{B} 1_{b}\right\rangle,\left|0_{a} 0_{B} 0_{b}\right\rangle \rightarrow\left|0_{a} 0_{B} 0_{b}\right\rangle$. The working mechanism and imperfections are detailed in Supplemental Material, Sec. G. The light fields emitted from nodes B and C are synchronously directed to a swapping station equipped with a 50/ 50 beamsplitter and two single-photon detectors, as illustrated in Fig. 2b. Conditioning on detecting a single photon allows for a probabilistic projection onto the state $\left|\Psi_{G H Z}\right\rangle=\left(\left|0_{A} 0_{B} 1_{C}\right\rangle+\left|1_{A} 1_{B} 0_{C}\right\rangle\right) / \sqrt{2}$. The path length fluctuation in fibers $A B$ and $C B$ can be solved by phase stabilization, similar to 1D repeater schemes. ${ }^{34}$ For example, it can be achieved by exciting memories in $\mathrm{A}$ and $\mathrm{C}$ in a Sagnac interferometer configuration, ${ }^{42,43}$ such that the excitation laser pulses for the two memories and the emitted photons travel the same path in a counter-propagating fashion.

For merging three $\mathrm{GHZ}$ states, the states of ensembles at adjacent nodes are projected onto the one-excitation subspace. This operation is realized by reading out the atomic excitations ${ }^{39-41}$ and directing the emitted light fields to the swapping station described above [see also inset of Fig. 2a], where success is heralded by the detection of a single photon (see Sec. A of Supplemental Material for details), otherwise the resulting state is discarded.

\section{Error filtering}

Apart from the major challenge to mitigate photon transmission losses, experimental limitations at the individual nodes have to be taken into account. The most important local error sources are the read-out inefficiency $v$ and imperfect photon detectors (with a detection inefficiency $f$ and a dark count probability $d$ during a photon pulse detection). Moreover, quantum states stored in the ensembles are degraded over time. Due to the encoding used here, the relevant physical mechanism - dephasing of individual emitters - leads to an effective decay of the stored collective excitations (Supplemental Material, Sec. F). In the considered ensemble-based setting, the main imperfections are therefore errors of loss type and detector dark counts.

The proposed 2D repeater protocol is designed to prevent the propagation of this kind of errors by employing an intrinsic redundancy of the native $2 \mathrm{D}$ network to filter out the errors in the merging process at each nesting level. The filtering mechanism, illustrated in Fig. 3, works as follows. At each nesting level one has to perform three merging operations to complete the entanglement generation between the three outermost nodes ( $X, Y, Z$ in Fig. 3). The third merging operation is redundant and it shows, if unsuccessful, that there is a lack or an excess of excitations in the generated state. This indicates an occurrence of either the loss type error or the multi-excitation error at previous stages of the protocol. In our protocol, the errors have, therefore, to conspire within one nesting level to pass undetected. In contrast, in the 1D benchmark scheme the errors can conspire within all levels of the bipartite state preparation to pass filtration in the last generation step. As a result, the $2 \mathrm{D}$ architecture exhibits a slower rate of error probabilities growth with the increase of the network nesting level (see Supplemental Material, Sec. A).

However, the filtering is a passive operation, therefore, it is important to start the entanglement distribution with initial states of high fidelity. As detailed in Sec. A and B of Supplemental Material, the protocol for generating entanglement at the 

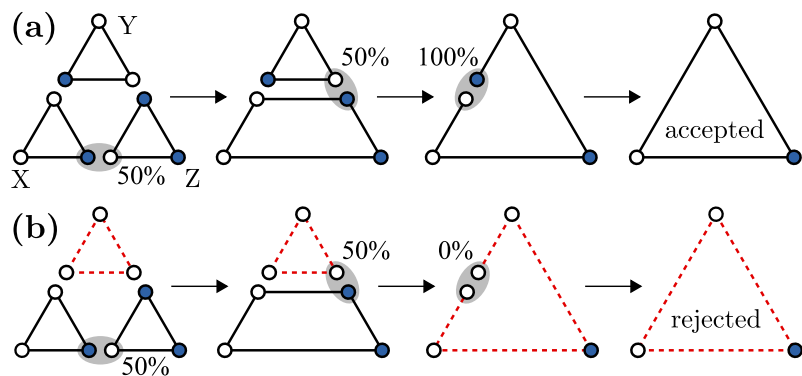

Fig. 3 Error filtering mechanism of the 2D repeater scheme. Solid (red dashed) lines represent quantum (classical) correlations of the states of the nodes shown with circles. The states of the nodes of the same (different) colors are correlated (anti-correlated). Merging operations are shown with gray ellipses, the corresponding success probabilities (neglecting merging imperfections) are indicated nearby. a The no errors case: two successful probabilistic mergings of the three constituent $\mathrm{GHZ}$ states are followed by the third deterministic merging producing a perfect $\mathrm{GHZ}$ state $\left(\left|0_{X} 0_{Y} 1_{Z}\right\rangle+\left|1_{X} 1_{Y} 0_{Z}\right\rangle\right) / \sqrt{2}$. b One loss type error or one multiexcitation error leading to preparation of a network segment in a wrong state (red triangle) can be deterministically filtered out.

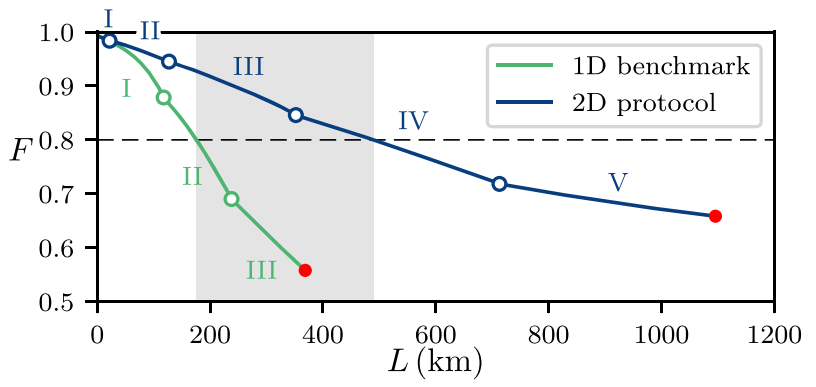

Fig. 4 Fidelity $F$ for the proposed 2D scheme and the 1D benchmark (see text) in the limit of infinite memory coherence time, as a function of the covered distance $L$. This plot corresponds to a cut of Fig. 1 at $T_{\text {coh }} \rightarrow \infty$. The gray area illustrates that for large distances, certain target fidelities can only be reached by the 2D scheme (the desired target fidelity depends on the application, in this example $F_{\text {target }}=0.8$ ). Roman numbers indicate the optimal number of nesting levels for the network. Transitions between the optimal numbers of levels are shown by circles. Red dots indicate the distance beyond which entanglement distillation is impossible.

elementary level and the relative orientation of $\mathrm{GHZ}$ states in the scheme is chosen such that the involved measurements allow for (i) identifying imperfect merging operations for ideal input states and (ii) filtering out imperfect states for ideal merging operations. The initial states of high fidelity at the elementary level and the subsequent error filtering at each nesting level result in the scheme robust against the vacuum and multi-excitation errors.

\section{Key features}

In the following, we highlight some of the main properties of our scheme and summarize the effect of limited memory coherence times in the presence of experimental imperfections. Figure 1a shows the fidelity of the generated state $\rho$ with respect to the target state $\left|\Psi_{\mathrm{GHZ}}\right\rangle, \quad F=\sqrt{\left\langle\Psi_{\mathrm{GHZ}}|\rho| \Psi_{\mathrm{GHZ}}\right\rangle}$, for the proposed architecture. In Fig. 1b, for comparison, we introduce a fidelity benchmark representing the performance of a 1D scheme based on long-distance bipartite entanglement distribution. The 1D benchmark uses conventional ensemble-based repeater scheme which creates three long-distance Bell pairs to be subsequently merged to generate the desired target state. The benchmark estimates an upper bound of the fidelity as ideal local GHZ states [shown with double lined triangles in the inset in Fig. 1b] are used for the final merging. In contrast to the 2D approach, which relies on multi-party entanglement at all network levels, the 1D scheme exploits the distribution of long-distance Bell pairs and involves the error filtering mechanism only in the end (Methods section).

The most striking feature of Fig. 1 is the fact that the 2D protocol can distribute $\mathrm{GHZ}$ states over increasingly large distances by increasing the memory coherence time (or alternatively, by using so-called multiplexing approaches ${ }^{44-48}$ in which several quantum memories or memory modes are used in parallel to compensate for limited coherence times). An increase of $T_{\text {coh }}$ significantly extends the distance that can be covered, since our protocol profits from using higher nesting levels for larger distances. This property makes the scheme practical and scalable. This is not the case for the 1D benchmark based on a regular DLCZ scheme, which is hampered by fundamental difficulties associated with the creation of long-range bipartite links (see Supplemental Material, Sec. A3 and Sec. C for details). Therefore, the 1D strategy reaches its performance limit at much shorter distances than the $2 \mathrm{D}$ scheme as can be seen in Fig. $1 \mathrm{~b}$ in comparison with Fig. $1 \mathrm{a}$.

To further characterize the potential of our approach, we analyze the case of infinite memory coherence time. Figure 4 shows the fidelity achievable by a network for which the number of nesting levels is optimal for a given distance in the presence of typical experimental imperfections for $T_{\text {coh }} \rightarrow \infty$. A network with more nesting levels divides the target distance into shorter segments what reduces the probability to lose photons during transmission at the cost of additional errors generated by the repeater scheme itself. For each target distance, there is an ideal number of nesting levels beyond which the addition of a repeater station becomes undesirable. The comparison of the 2D approach (black line) with the 1D benchmark (green line) for generating three-party $\mathrm{GHZ}$ states shows that the latter suffers from severe limitations in the achievable fidelities for large distances even for perfect quantum memories. This is the result of the faster errors accumulation with the growing network size in 1D scheme which lacks the filtering mechanism of the native 2D protocol, as discussed in the previous section.

Here we emphasize that although the 1D benchmark uses fewer resources (memory cells) than our 2D scheme (see Supplemental Material Sec. A2) its performance is saturated at much shorter distances and can not be improved by a simple increase of resources invested into multiplexing. The presented 2D architecture allows us to convert the extra resources into longer entanglement distribution distances with higher target fidelities. Moreover, the additional memory cells improve coverage of intrinsically 2D networks by allowing the multi-party entanglement generation between arbitrary nodes of the network (see Supplemental Material Sec. A1), while 1D based schemes entangle only the outermost ensembles.

A detailed analysis of the 2D protocol performance, including an assessment of the achievable rates, is provided in Supplemental Material (Sec. A3 and Sec. C). In the following, we discuss means to further improve the performance by temporal filtering.

\section{Temporal filtering protocol}

Limited coherence times of quantum memories are generally an important restricting factor for quantum repeaters. To mitigate their effect, we introduce a temporal filtering mechanism by defining a time window $\tau$, after which quantum states are discarded. During the probabilistic generation and merging procedure shown in Fig. 2, ensembles storing excitations longer than $\tau$ are reset to their ground state, such that the entanglement generation process involving these nodes starts anew. As a result, the influence of decoherence is decreased at the expense of a reduced rate. The time $\tau$ can be changed dynamically by the control software without changing the hardware in a quantum 


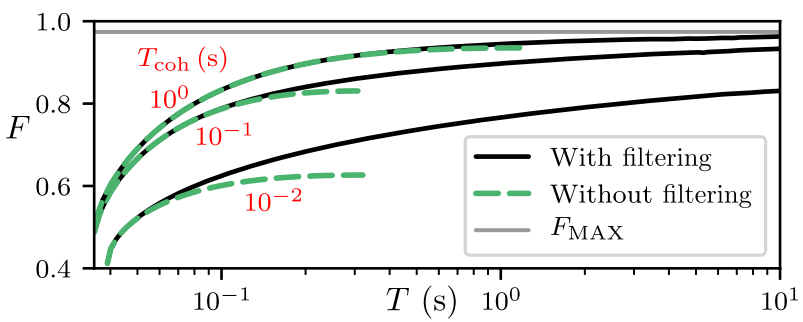

Fig. 5 Temporal filtering for a 2D network with nesting level I, i.e. 9 ensembles [see Fig. 2b] for the parameters specified in Methods section. The fidelity $F$ is shown versus the entanglement generation time $T$ for a target distance $L=50 \mathrm{~km}$ and different memory times $T_{\text {coh }}=\left\{10^{-2}, 10^{-1}, 1\right\} \mathrm{s}$. Black solid (dashed green) lines represent results with (without) temporal filtering. The gray solid line indicates the fidelity in the limit of infinite quantum memory times.

network. Due to this added flexibility, different types of applications - requiring either high fidelities or high rates can be accommodated. The corresponding tradeoff has been calculated semi-analytically (as described below) and is shown in Fig. 5.

\section{Diagrammatic technique}

We used a numerical Monte Carlo algorithm and developed a new semi-analytical technique to analyze the performance of our scheme in the presence of realistic imperfections. The Monte Carlo method is very flexible and simulates the full repeater protocol step by step. ${ }^{49,50}$ The strength of this exact simulation is also its weakness - the runtime is proportional to the entanglement generation time in the quantum network, growing quickly with the network's scale.

The analytical method overcomes this difficulty while still incorporating all relevant error sources, including finite memory coherence times. It takes time delays due to classical communication into account and can be used to analyze a large class of repeater schemes, including $1 \mathrm{D}$ protocols. The main idea is to determine the density matrix distribution $\varrho(t)$ for the ensemble of states generated by the network at time $t$. More precisely, we obtain the Laplace image of this distribution, $\tilde{\varrho}(s)$, which fully describes the statistics of the network and allows one to infer the average generated state $\rho \equiv \int_{0}^{\infty} \varrho(t) \mathrm{d} t=\left.\tilde{\varrho}(s)\right|_{s=0}$, corresponding generation time $T \equiv \int_{0}^{\infty} t \operatorname{Tr} \varrho(t) \mathrm{d} t=-\left.\frac{d}{d s} \operatorname{Tr} \tilde{Q}(s)\right|_{s=0}$, and other relevant statistical quantities.

We assume that the probability $p_{1}$ to generate an entangled state of an elementary segment is small for each time step $\Delta t$, such that the continuous probability density $p(t) \equiv \nu e^{-\nu t}$ with the rate $\nu \equiv p_{1} / \Delta t$ can be introduced. As an example, we consider a linear network consisting of two links, where the density matrix distribution for generating the $a^{\text {th }}$ link in a state $\rho_{a}$ before the $\beta^{\text {th }}$ link has been generated at time $t$ in a state $\rho_{\beta}$ is given by $\varrho_{a \beta}(t)=p_{\beta}(t) \int_{0}^{t} \mathrm{~d} t^{\prime} p_{a}\left(t^{\prime}\right) e^{\left(t-t^{\prime}\right) \mathcal{L}_{a}} \rho_{1} \otimes \rho_{2}$ with $a \beta \in\{12,21\}$. Here we sum over all intermediate times $t^{\prime}$ of the $a^{\text {th }}$ link creation. The degradation of the link during the waiting time $t-t^{\prime}$ due to finite memory life times is taken into account using the superoperator $\mathcal{L}_{\alpha}$. The corresponding Laplace image of the distribution is given by $\tilde{\varrho}_{a \beta}(s)=\nu_{a} \nu_{\beta} /\left.\left[\left(s^{\prime}+\nu_{\alpha}\right)\left(s^{\prime}-\mathcal{L}_{\alpha}\right)\right] \cdot \rho_{1} \otimes \rho_{2}\right|_{s^{\prime}=s+\nu_{\beta}}$.

The probability to generate and successfully merge states of two segments during the time window $[t, t+d t)$ is given by $\operatorname{Tr} \mathcal{M} \varrho_{a \beta}(t) d t$, with $\mathcal{M}$ the merging superoperator. The summation over all possible combinations of unsuccessful mergings leading to the generation of an entangled state results in a sum of multiple convolutions in the time domain. In the Laplace domain, the sum converges to

$\tilde{\varrho}(s)=\frac{\sum_{a \beta} \mathcal{M} \tilde{\varrho}_{\alpha \beta}(s)}{1-\sum_{a \beta} \operatorname{Tr}(\mathcal{I}-\mathcal{M}) \tilde{\varrho}_{\alpha \beta}(s)}$, with $\sum_{\alpha \beta}$ the sum over all possible orders in which links are generated and $\mathcal{I}$ the unit superoperator. The Laplace image (1) is used to find the average density matrix $\rho^{\prime}$ and generation time $T^{\prime}$ for the given nesting level of the network, as described above. To address the next network level, we apply the approximation that the segments are generated time-independently in the state $\rho^{\prime}$ with the rate $\nu^{\prime}=1 / T^{\prime}$. In a recursive procedure, the state $\rho$ and generation time $T$ of an arbitrary network level can be found. As detailed in Sec. E of Supplemental Material, we introduce diagrams to conveniently deal with probabilistic processes in networks of arbitrary complexity.

\section{DISCUSSION}

We proposed a scalable 2D architecture for generating longdistance multi-party entanglement and provided a full performance analysis with emphasis on the effect of finite memory lifetimes. Covering increasingly longer distances as shown in Fig. 1 can be achieved either using memories with long coherence times or using multiplexing approaches, ${ }^{44-48}$ in which several memorymodes are used in parallel. The presented scheme provides a flexible structure for creating GHZ states between arbitrary nodes of the 2D network, not only between the outermost ensembles. The logical topology of the network [shown in Fig. 2a] is thereby not identical to the topology of the required fiber links, which can easily be adjusted to accommodate urban constraints. We are here primarily interested in metropolitan distances and applications requiring only moderate bit-rates. Important examples include secret voting and the protection of classified information that requires several parties for decryption. ${ }^{26,27}$

The presented scheme can be modified to work without cavities at the expense of using a larger number of ensembles (see Supplemental Material, Sec. H). This modification uses polarization-type qubits ${ }^{34,39}$ and two-click conditioning, ${ }^{51}$ enhancing its resistance with respect to fiber-length fluctuations. It will be interesting to develop similarly robust schemes for other platforms such as trapped ions, ${ }^{8,52}$ in which a universal gate set can be implemented. In this case, more complicated 2D repeater protocols involving quantum error correction ${ }^{33}$ instead of error filtering could be envisaged.

\section{METHODS}

Parameters of the simulated network

The results of simulations presented in Figs 1, 4, and 5 are obtained with the following parameters of the network: detection inefficiency $f=0.05$, read-out inefficiencies $v=0.05$, fiber attenuation length $L_{\text {att }}=22 \mathrm{~km}$, gate efficiency (see text) $\eta=0.6$, signal length $I=10^{-4} \mathrm{~s}$, and dark count probability in the signal measurement operation $d=$ $10 \mathrm{~Hz} \times 10^{-4} \mathrm{~s}=0.001$.

\section{One-dimensional benchmark scheme}

Here we present an alternative, one-dimensional (1D), repeater approach for distribution of multi-partite GHZ state, which provides us with the fidelity benchmark used in the present work. This $1 \mathrm{D}$ benchmark scheme is based on the generation of long-range bipartite quantum states that are created using regular DLCZ quantum repeaters. A comparison of various nesting levels structures for the 1D benchmark scheme and the propose 2D scheme is presented in Fig. 6 a.

While two links are enough to distribute entanglement between three parties, we equip the 1D scheme with the third link, which is used to implement error filtering procedure in the final step, similar to the original DLCZ protocol, as explained below. To ensure the robustness of the benchmark we assume that the distant parties $X, Y$, and $Z$ of the 1D scheme have access to ideal GHZ states that can be generated on demand. This provides an upper bound on the performance of $1 D$ schemes of DLCZ type for distribution of tripartite GHZ states. Therefore, the corresponding fidelity of the 1D scheme serves as a benchmark for our 2D scheme. 


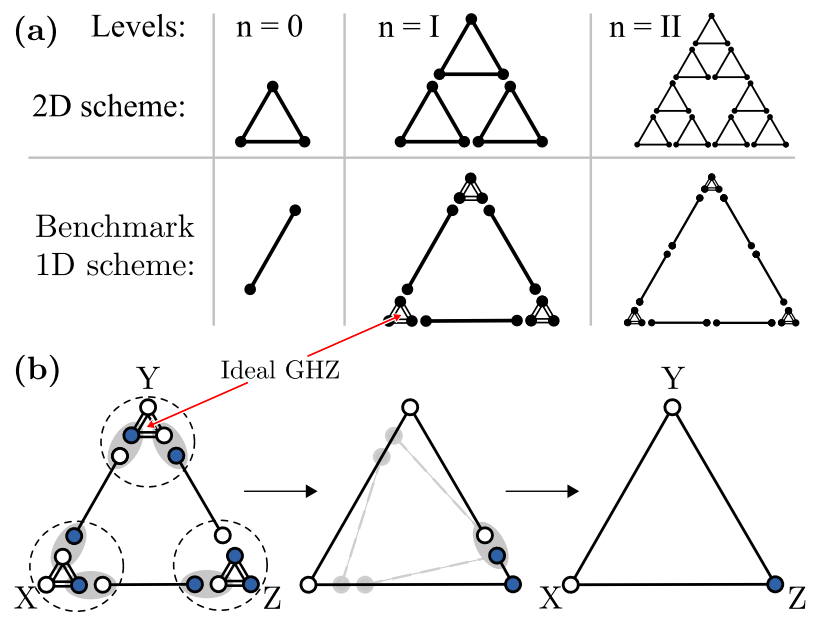

Fig. 6 One-dimensional benchmark scheme. a Comparison of the 2D scheme and the 1D benchmark scheme at different nesting levels. Dots represent quantum memories. Dots connected by lines represent elementary segments of the networks. Triangles with double lines indicate ideal GHZ states. b The last step of generating $\left|\Psi_{\mathrm{GHZ}}\right\rangle$ in the 1D benchmark scheme. The pictorial representation of states is explained in Fig. 3. The three distant parties X, Y, and Z hold perfect tripartite $\mathrm{GHZ}$ states, represented by triangles. The final filtering step described in the text is depicted in two stages, shown by arrows. Gray dashed lines and circles indicate the actual successive merging pattern, during which the long-range quantum links are first merged with the local GHZ states and then with each other.

Figure $6 \mathrm{~b}$ shows the final merging step of the 1D scheme acting as an error filtering operation. In this step, five swapping operations are followed by the last one, which is deterministic in the ideal case. A failure of the last swapping operation indicates an erroneous state. More details about the 1D benchmark scheme are presented in Sec. A of Supplemental Material.

Note added

Recently we have become aware of Santra et al. ${ }^{53}$ discussing a similar temporal filtering technique

\section{DATA AVAILABILITY}

The data that support the findings of this study are available from the corresponding author upon reasonable request.

\section{CODE AVAILABILITY}

The code that supports the findings of this study are available from the corresponding author upon reasonable request.

Received: 22 May 2019; Accepted: 25 November 2019; Published online: 20 December 2019

\section{REFERENCES}

1. Bruß, D. \& Lütkenhaus, N. Quantum key distribution: from principles to practicalities. Appl. Algebra Eng. Commun. Comput. 10, 383-399 (2000).

2. Beals, R. et al. Efficient distributed quantum computing. Proc. Math. Phys. Eng. Sci. 469, 20120686 (2013).

3. Komar, P. et al. A quantum network of clocks. Nat. Phys. 10, 582-587 (2014).

4. Eldredge, Z., Foss-Feig, M., Gross, J. A., Rolston, S. L. \& Gorshkov, A. V. Optimal and secure measurement protocols for quantum sensor networks. Phys. Rev. A 97, 042337 (2018).

5. Proctor, T. J., Knott, P. A. \& Dunningham, J. A. Networked quantum sensing. Preprint at https://arxiv.org/abs/1702.04271 (2017).
6. Ge, W., Jacobs, K., Eldredge, Z., Gorshkov, A. V. \& Foss-Feig, M. Distributed quantum metrology with linear networks and separable inputs. Phys. Rev. Lett. 121, 043604 (2018).

7. Ritter, S. et al. An elementary quantum network of single atoms in optical cavities Nature 484, 195-200 (2012).

8. Northup, T. E. \& Blatt, R. Quantum information transfer using photons. Nat Photon. 8, 356-363 (2014).

9. Chou, C. W. et al. Measurement-induced entanglement for excitation stored in remote atomic ensembles. Nature 438, 828-832 (2005).

10. Yuan, Z. S. et al. Experimental demonstration of a BDCZ quantum repeater node. Nature 454, 1098-1101 (2008).

11. Olmschenk, S. et al. Quantum teleportation between distant matter qubits. Science 323, 486 (2009).

12. Lee, K. C. et al. Entangling macroscopic diamonds at room temperature. Science 334, 1253-1256 (2011).

13. Krauter, $\mathrm{H}$. et al. Deterministic quantum teleportation between distant atomic objects. Nat. Phys. 9, 400-404 (2013).

14. Pfaff, W. et al. Unconditional quantum teleportation between distant solid-state quantum bits. Science 345, 532 (2014).

15. Delteil, A. et al. Generation of heralded entanglement between distant hole spins. Nat. Phys. 12, 218-223 (2016).

16. Hofmann, J. et al. Heralded entanglement between widely separated atoms. Science 337, 72 (2012).

17. Hensen, B. et al. Loophole-free bell inequality violation using electron spins separated by 1.3 kilometres. Nature 526, 682-686 (2015).

18. Epping, M., Kampermann, H. \& Bruß, D. Large-scale quantum networks based on graphs. New J. Phys. 18, 053036 (2016).

19. Kimble, H. J. The quantum internet. Nature 453, 1023-1030 (2008).

20. Li, Y., Cavalcanti, D. \& Kwek, L. C. Long-distance entanglement generation with scalable and robust two-dimensional quantum network. Phys. Rev. A 85, 062330 (2012).

21. Pant, M. et al. Routing entanglement in the quantum internet. npj Quantum Inf. 5, 25 (2019).

22. Das, S., Khatri, S. \& Dowling, J. P. Robust quantum network architectures and topologies for entanglement distribution. Phys. Rev. A 97, 012335 (2018)

23. Wehner, S., Elkouss, D. \& Hanson, R. Quantum internet: a vision for the road ahead. Science 362, eaam9288 (2018).

24. Pirandola S. Capacities of repeater-assisted quantum communications. Preprint at https://arxiv.org/abs/1601.00966 (2016).

25. Greenberger, D. M, Horne, M. \& Zeilinger, A. Bell's Theorem, Quantum Theory And Conceptions Of The Universe. Vol. 37 (Springer Netherlands, Dordrecht, 1989).

26. Hillery, M., Ziman, M., Buzek, V. \& Bielikova, M. Towards quantum-based privacy and voting. Phys. Lett. A 349, 75-81 (2006).

27. Chen, X.-B., Niu, X.-X., Zhou, X.-J. \& Yang, Y.-X. Multi-party quantum secret sharing with the single-particle quantum state to encode the information. Quantum Inf. Process. 12, 365-380 (2013).

28. Thapliyal, K., Pathak, A. \& Sharma, R. D. Protocols for quantum binary voting. Int. J. Quantum Inf. 15, 1750007 (2017).

29. Mermin, N. D. Quantum mysteries revisited. Am. J. Phys. 58, 731-734 (1990).

30. Takeoka, M., Guha, S. \& Wilde, M. M. Fundamental rate-loss tradeoff for optical quantum key distribution. Nat. Commun. 5, 5235 (2014).

31. Pirandola, S., Laurenza, R., Ottaviani, C. \& Banchi, L. Fundamental limits of repeaterless quantum communications. Nat. Commun. 8, 15043 (2017).

32. Briegel, H.-J., Dür, W., Cirac, J. I. \& Zoller, P. Quantum repeaters: the role of imperfect local operations in quantum communication. Phys. Rev. Lett. 81, 5932-5935 (1998).

33. Muralidharan, S. et al. Optimal architectures for long distance quantum communication. Sci. Rep. 6, 20463 (2016).

34. Sangouard, N., Simon, C., De Riedmatten, H. \& Gisin, N. Quantum repeaters based on atomic ensembles and linear optics. Rev. Modern Phys. 83, 33-80 (2011).

35. Wallnöfer, J., Zwerger, M., Muschik, C., Sangouard, N. \& Dür, W. Two-dimensional quantum repeaters. Phys. Rev. A 94, 052307 (2016).

36. Riedmatten, de, H. \& Afzelius, M. Quantum Light Storage in Solid State Atomic Ensembles. p. 241-273 (Springer International Publishing, Cham, 2015).

37. Simon, C. et al. Quantum memories - a review based on the european integrated project "qubit applications (qap)". Eur. Phys. J. D 58, 1-22 (2010)

38. Zhong, M. et al. Optically addressable nuclear spins in a solid with a six-hour coherence time. Nature 517, 177-180 (2015).

39. Duan, L.-M., Lukin, M., Cirac, I. \& Zoller, P. Long-distance quantum communication with atomic ensembles and linear optics. Nature 414, 413-418 (2001).

40. Simon, J., Tanji, H., Thompson, J. K. \& Vuletić, V. Interfacing collective atomic excitations and single photons. Phys. Rev. Lett. 98, 183601 (2007).

41. Gorshkov, A. V., André, A., Lukin, M. D. \& Sørensen, A. S. Photon storage in $\Lambda$-type optically dense atomic media. ii. free-space model. Phys. Rev. A 76, 033805 (2007) 
42. Childress, L., Taylor, J. M., Sørensen, A. S. \& Lukin, M. D. Fault-tolerant quantum repeaters with minimal physical resources and implementations based on singlephoton emitters. Phys. Rev. A At. Mol. Opt. Phys. 72, 1-16 (2005).

43. Mináŕ, J., De Riedmatten, H., Simon, C., Zbinden, H. \& Gisin, N. Phase-noise measurements in long-fiber interferometers for quantum-repeater applications. Phys. Rev. A At. Mol. Opt. Phys. 77, 1-8 (2008).

44. Collins, O. A., Jenkins, S. D., Kuzmich, A. \& Kennedy, T. A. B. Multiplexed memoryinsensitive quantum repeaters. Phys. Rev. Lett. 98, 060502 (2007).

45. Simon, C. et al. Quantum repeaters with photon pair sources and multimode memories. Phys. Rev. Lett. 98, 190503 (2007).

46. Bonarota, M., Le Gouët, J.-L. \& Chanelière, T. Highly multimode storage in a crystal. New J. Phys 13, 013013 (2011).

47. Abruzzo, S., Kampermann, H. \& Bruß, D. Finite-range multiplexing enhances quantum key distribution via quantum repeaters. Phys. Rev. A 89, 012303 (2014).

48. Suzanne B, vD., Peter C, H., Filip, R., Stephanie, W. \& Ronald, H. Multiplexed entanglement generation over quantum networks using multi-qubit nodes. Quant. Sci. Technol. 2, 034002 (2017).

49. Jones, C., Kim, D., Rakher, M. T., Kwiat, P. G. \& Ladd, T. D. Design and analysis of communication protocols for quantum repeater networks. New J. Phys. 18, 083015 (2016).

50. Van Meter, R., Satoh, T., Nagayama, S., Matsuo, T. \& Suzuki, S. Optimizing timing of high-success-probability quantum repeaters. Preprint at https://arxiv.org/abs/ 1701.04586 (2017).

51. Sangouard, N. et al. Robust and efficient quantum repeaters with atomic ensembles and linear optics. Phys. Rev. A 77, 062301 (2008).

52. Duan, L. M. \& Monroe, C. Colloquium: quantum networks with trapped ions. Rev. Mod. Phys. 82, 1209-1224 (2010).

53. Santra, S., Jiang, L. \& Malinovsky, V. S. Quantum repeater architecture with hierarchically optimized memory buffer times. Quant. Sci. Technol. 4, 025010 (2019).

\section{ACKNOWLEDGEMENTS}

We thank M. Afzelius and P. Jobez for fruitful discussions on solid-state ensemblebased implementations and J. Wallnöfer for his input on 2D architectures. Research was sponsored by the Swiss National Foundation (SNSF) through grant number PP00P2-179109, by the Austrian Science Fund (FWF): P28000-N27, P30937-N27, and by the Army Research Laboratory and was accomplished under Cooperative Agreement Number W911NF-15-2-0060. The views and conclusions contained in this document are those of the authors and should not be interpreted as representing the official policies, either expressed or implied, of the Army Research Laboratory or the U.S. Government. The U.S. Government is authorized to reproduce and distribute reprints for Government purposes notwithstanding any copyright notation herein. This research was undertaken thanks in part to funding from CFREF.

\section{AUTHOR CONTRIBUTIONS}

All authors contributed equally to the current paper.

\section{COMPETING INTERESTS}

The authors declare no competing interests.

\section{ADDITIONAL INFORMATION}

Supplementary information is available for this paper at https://doi.org/10.1038/ s41534-019-0230-3.

Correspondence and requests for materials should be addressed to V.V.K.

Reprints and permission information is available at http://www.nature.com/ reprints

Publisher's note Springer Nature remains neutral with regard to jurisdictional claims in published maps and institutional affiliations.

\section{cc) (i)}

Open Access This article is licensed under a Creative Commons Attribution 4.0 International License, which permits use, sharing, adaptation, distribution and reproduction in any medium or format, as long as you give appropriate credit to the original author(s) and the source, provide a link to the Creative Commons license, and indicate if changes were made. The images or other third party material in this article are included in the article's Creative Commons license, unless indicated otherwise in a credit line to the material. If material is not included in the article's Creative Commons license and your intended use is not permitted by statutory regulation or exceeds the permitted use, you will need to obtain permission directly from the copyright holder. To view a copy of this license, visit http://creativecommons. org/licenses/by/4.0/.

(c) The Author(s) 2019 